\title{
ANÁLISE COMPARATIVA DE PROTOCOLOS FIELDBUS (WIRELESSHART, PROFIBUS E FOUDATION FIELDBUS)*
}

Diego Linhares de Azevedo ${ }^{1}$ Fábio Ferreira da Silva ${ }^{2}$ Luiz Montoio Rivelinir ${ }^{3}$ Mauricio Toledo ${ }^{4}$ Paulo Dotti ${ }^{3}$

\section{Resumo}

Este artigo contém uma pequena introdução à alguns dos protocolos de redes industriais mais populares atualmente, Foundation Fieldbus, Profibus e WirelessHart, apresentando brevemente suas arquiteturas e características, e, então, realizando uma análise comparativa de suas vantagens e desvantagens.

Palavras-chave: Redes industriais; Profibus; FF; Foudation fieldbus; WirelessHart; Análise qualitativa; Comparação.

\section{COMPARATIVE ANALYSIS OF FIELDBUS PROTOCOLS (WIRELESSHART, PROFIBUS AND FOUNDATION FIELDBUS}

\section{Abstract}

This article contains an introduction to some of the most popular industrial fieldbus protocols nowadays, Foudation Fieldbus, Profibus and WirelessHart, presenting briefly each architecture and caracteristics, and comparatively analysing their advantages and disadvantages.

Keywords: Fieldbuses; Fieldbus; Profibus; FF; Foudation Fieldbus; WirelessHart; Qualitative analysis.

1 Bacharel em Engenharia Elétrica pela UFCG, Engenheiro de Projetos na ABB, Sorocaba, SP, Brasil.

2 Bacharel em Engenharia Elétrica pela UFCG, Engenheiro de Projetos na ABB, Osasco, SP, Brasil.

3 Bacharel em Engenharia Elétrica pela Unesp, Engenheiro de Projetos na ABB- Sorocaba, SP, Brasil.

4 Bacharel em Engenharia Elétrica pela Unicamp, Engenheiro de Projetos na ABB-Sorocaba, SP, Brasil. 


\section{INTRODUÇÃO}

O desenvolvimento da indústria de Automação está diretamente ligado a necessidade dos meios produtivos de incrementar a sua eficiência através da economia de energia, força de trabalho e matéria-prima e incremento de produtividade e segurança nas operações.

Em meados da década de 1940, surgiram os sistemas de controle de processo, utilizando basicamente tecnologia pneumática e mecânica. Com a evolução da eletrônica, os sistemas baseados em sinais elétricos/analógicos ganharam grande usabilidade na década de sessenta.

Com a popularização do uso da microeletrônica no final da década de setenta, foi possível migrar para uma arquitetura digital.

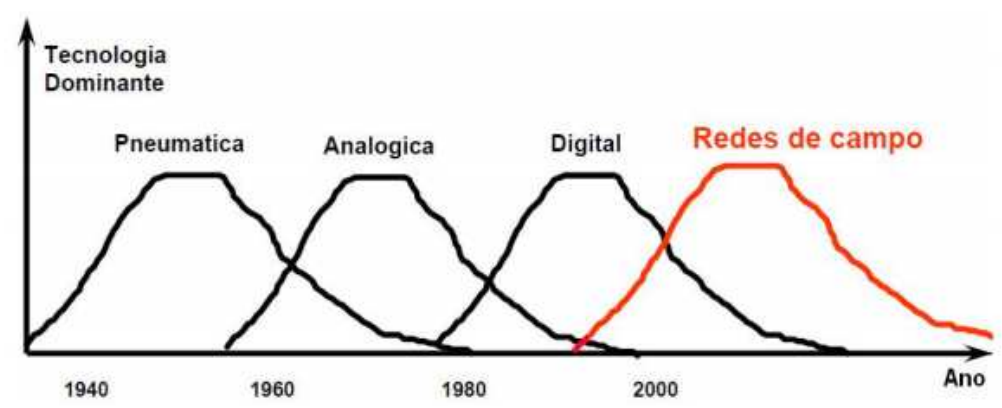

Figura 1- Evolutivo da tecnologia de automação dominante [1].

Nos anos oitenta, surgiram diversos protocolos industriais, proprietários na sua maioria - não se falavam, que deram origem as redes de campo.

Para tentar possibilitar a interoperabilidade entre esses protocolos, foram realizados alguns esforços no intuito de definir padrões que garantissem a comunicação entre sistemas heterogêneos, sendo o mais famoso o modelo OSI ${ }^{1}$.

Dentre os patrões existentes atualmente no mercado podemos destacar os Profibus, Fieldbus Foundation e o WirelessHART.

Neste trabalho iremos realizar uma análise comparativa entre os mesmos.

\section{PROFIBUS}

Desde a década de setenta existem sistemas "fieldbus" instalados, mas eram na sua maioria prioritários, ou seja, "não se falavam". Em meados da década de oitenta a comunidade europeia decidiu iniciar um projeto com o intuito de obter um padrão de barramento bitserial estável, aberto a diversos fabricantes, que atendesse a necessidade dos usuários de equipamentos de automação de processos.

Tal iniciativa foi chamada de Profibus e tendo a participação de vinte e uma instituições e empresas.

O Profibus é composto por quatro definições, sendo elas:

- Profibus DP (Decentralized Periphery);

- Profibus FMS (Fieldbus Message Specification);

- Profibus PA (Process Automation);

- Profinet.

\footnotetext{
${ }^{1}$ OSI - (criado em 1970 e formalizado em 1983) é um modelo de referência da ISO que possui como principal objetivo ser padrão para protocolos de comunicação entre soluções heterogêneas, garantindo assim a comunicação end-to-end [2].
} 
A arquitetura do protocolo é baseada no modelo OSI, onde a camada 1 (nível físico) define as características físicas de transmissão, a camada 2 (enlace de dados) o protocolo de acesso ao meio e a camada 7 (aplicação - somente para o FMS) define as funções de aplicação.

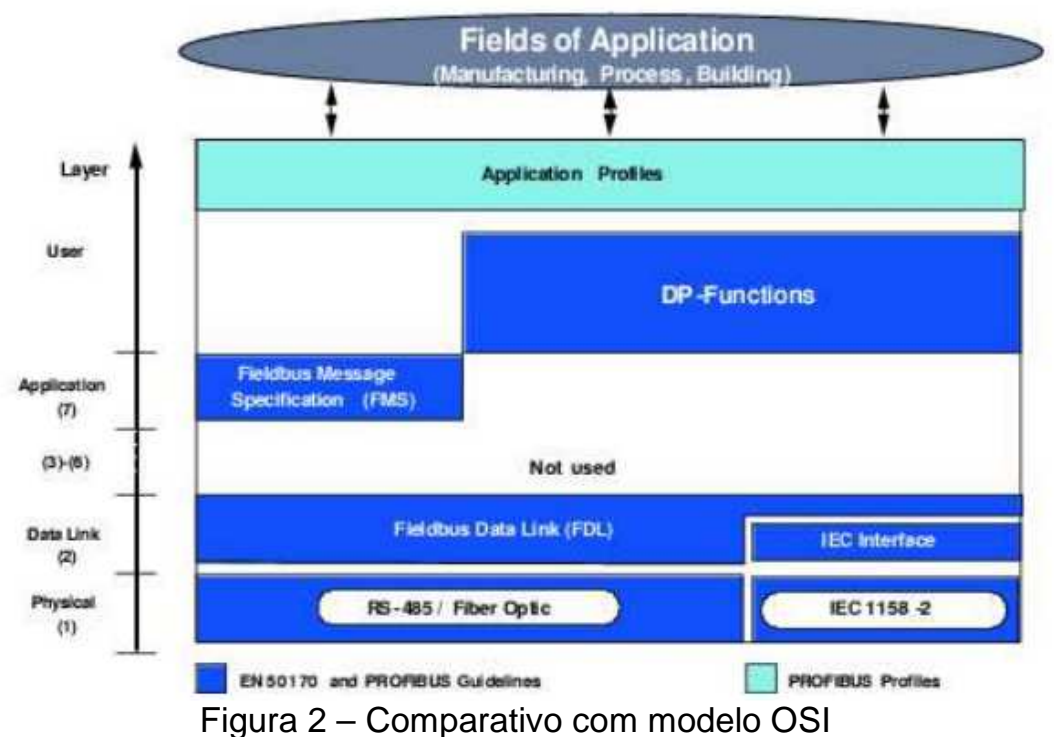

A rede Profibus é uma rede do tipo multidrop, assíncrona, half duplex e utiliza a comunicação do tipo passagem de token (Token Passing) e mestre-escravo. $O$ mecanismo de passagem de token permite a aplicação de múltiplos mestres em uma mesma rede compartilhando o acesso. Somente o mestre pode iniciar a comunicação na rede. Os escravos comunicam somente para responder requisições do mestre. A rede Profibus DP permite a operação permanente com mais de um mestre, desde que configurados individualmente e de maneira adequada nas restrições da norma [3].

Como citado acima, existem quatro tipos de dispositivos em uma rede profibus [4]:

- Mestre DP Classe 1: Responsável pelas operações cíclicas (leitura/escrita) e controle das malhas abertas e fechadas do sistema de controle/automação (CLP);

- Mestre DP Classe 2: Responsável pelos acessos acíclicos dos parâmetros e funções dos equipamentos PA (estação de engenharia ou estação de operação;

- Escravo - é um dispositivo periférico, tal como: dispositivos de $1 / 0$, atuadores, IHM, válvulas, transdutores, dispositivos que têm somente entrada, somente saída ou uma combinação de entradas e saídas. Aqui, ainda pode-se citar os escravos PA, uma vez que são vistos pelo sistema como se fossem escravos DP. A quantidade de informação de entrada e saída depende do tipo de dispositivo, sendo que se permite até 244 bytes de entrada e 244 bytes de saída;

- Acopladores - São dispositivos utilizados para traduzir as características físicas entre o PROFIBUS DP e o PROFIBUS PA (H1: 31,25 kbits/s). 


\section{WIRELESSHART}

O WirelessHart é um protocolo padrão sem fio, bastante robusto, que atende toda gama de processos de medição, controle e aplicações de gestão de ativos. Baseado no protocolo Hart, o WirelessHart permite aos usuários de forma rápida e fácil ganhar os benefícios da tecnologia sem fios, mantendo a compatibilidade com dispositivos, instrumentos de campo e ferramentas Hart [5].

Sua arquitetura é baseada no modelo de camada OSI, utilizando cinco das sete camadas. Como pode ser observado na figura 03.

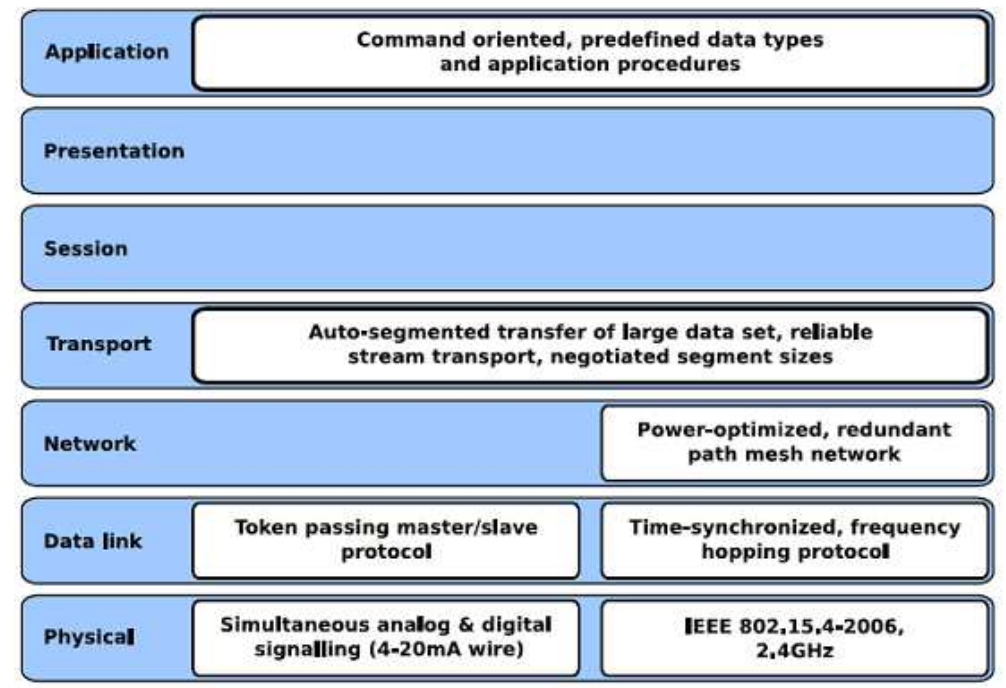

HART

WirelessHART

Figura 3 - Comparativo com modelo OSI (WirelessHART)

A camada física foi adaptada para operar conforme o padrão IEEE 802.15.4 em 2.4GHz ISM utilizando TDMA. Na camada de enlace tivemos o acréscimo foram acrescentados os protocolos "Frequence Hopping" e "Time-sinchronized".

Comparativamente ao Hart padrão, temos a inclusão da camada de rede permitindo assim o uso da topologia Mesh.

Nas camadas de aplicação e transporte o HART convencional e o WirelessHART possuem características compatíveis.

A rede WirelessHART geralmente é composta por um Gateway, Instrumentos WirelessHART e um Gerente de Rede.

- O Gerente de rede possui por finalidade gerenciar a rede Mesh e os instrumentos de rede. É responsável por distribuir as chaves de segurança, configurar e coordenar os instrumentos e a rede, como programação das comunicações entre os dispositivos, gestão da mensagem e rotas, além do monitoramento do bom desempenho da rede. O Network Manager é único por rede Mesh e pode ser integrado no gateway, no host ou no controlador do processo da automação [6];

- O gateway conecta à rede WirelessHART com a rede de automação da planta instalada. Ele fornece o acesso dos dispositivos de campo com o gerenciador da rede. Também possui a função de fazer a interpretação de protocolos de diferentes redes [7];

- Instrumentos WirelessHART - coletam as informações de processo e transmitem através da rede. 


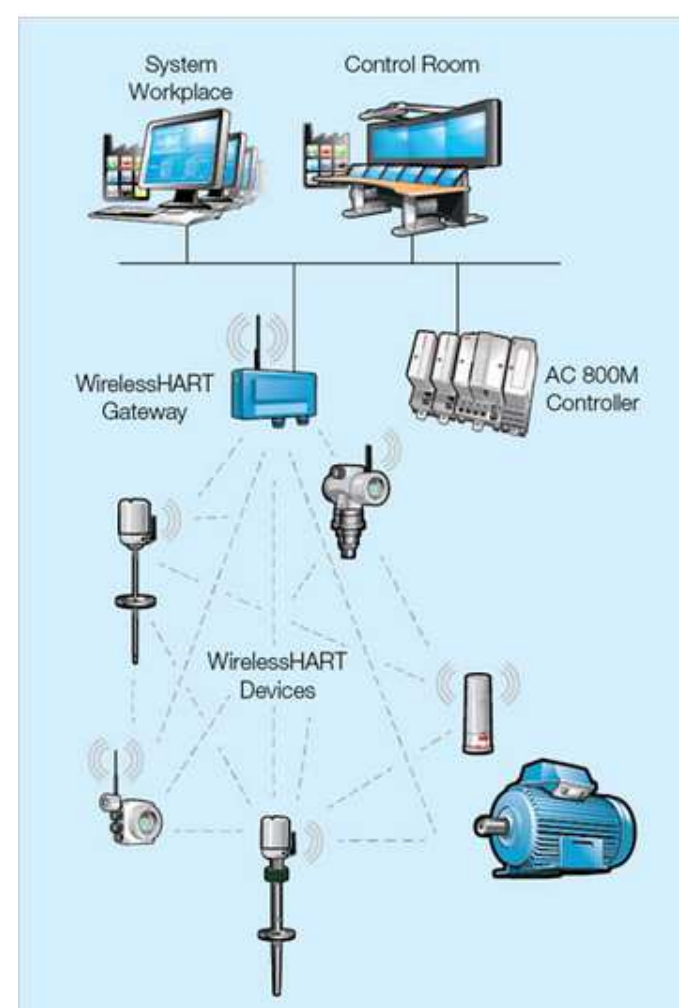

Figura 4 - Exemplo de rede MESH com WirelessHART usando infraestrutura ABB

Devido a característica da rede MESH, os instrumentos de campo podem servir como repetidores, provendo assim diversos possíveis trajetos para a comunicação. Recomenda-se que cada equipamento tenha visada para no mínimo três outros.

\section{FF}

O protocolo Fieldbus também foi desenvolvido baseado no padrão ISO/OSI embora não contenha todos os seus níveis. Pode-se em primeira análise dividi-lo em nível físico ("Physical Layer" - que trata das técnicas de interligação dos instrumentos) e níveis de software ("Communication Stack") que tratam da comunicação digital entre os equipamentos. A transmissão física é feita com corrente de $10 \mathrm{~mA}$ a $31,25 \mathrm{kbit} / \mathrm{s}$ com carga equivalente a $50 \mathrm{ohm}$.

O Fieldbus Foundation definiu um padrão para Aplicações de Usuário baseados em blocos. Os blocos descrevem características do dispositivo Fieldbus como nome, fabricante e número serial. Os parâmetros de entrada e saída $(\mathrm{I} / \mathrm{O})$ das FB podem ser acessados através da rede e a execução de cada função é precisamente agendada. As primeiras versões da norma especificam duas opções para a camada física: $\mathrm{H} 1$ e H2. $\mathrm{O} \mathrm{H} 1$, com taxa de $31.25 \mathrm{Kbits} / \mathrm{s}$ é voltado basicamente para equipamentos de campo (transmissores, posicionadores de válvula, etc), e pode ser usado em áreas onde é necessária segurança intrínseca (ambientes explosivos). 0 $\mathrm{H} 2$, com taxa de 1 a $2.5 \mathrm{Mbps}$, seria utilizado para integrar controladores e equipamentos mais complexos. Devido à rápida evolução tecnológica, o H2 foi substituído pelo HSE, que usa Ethernet a $100 \mathrm{Mbps}$. Assim, para conexão de equipamentos de campo há o FOUNDATIONTM Fieldbus H1, com camada física baseada na ISAS50.02-1992 ou IEC61158-2:2000. Para conexão entre PLCs, Linking Devices, Gateways e PCs, há o FOUNDATIONTM Fieldbus HSE, baseado em Ethernet (IEEE802.3-2000, ISO/IEC8802.3-2000). 


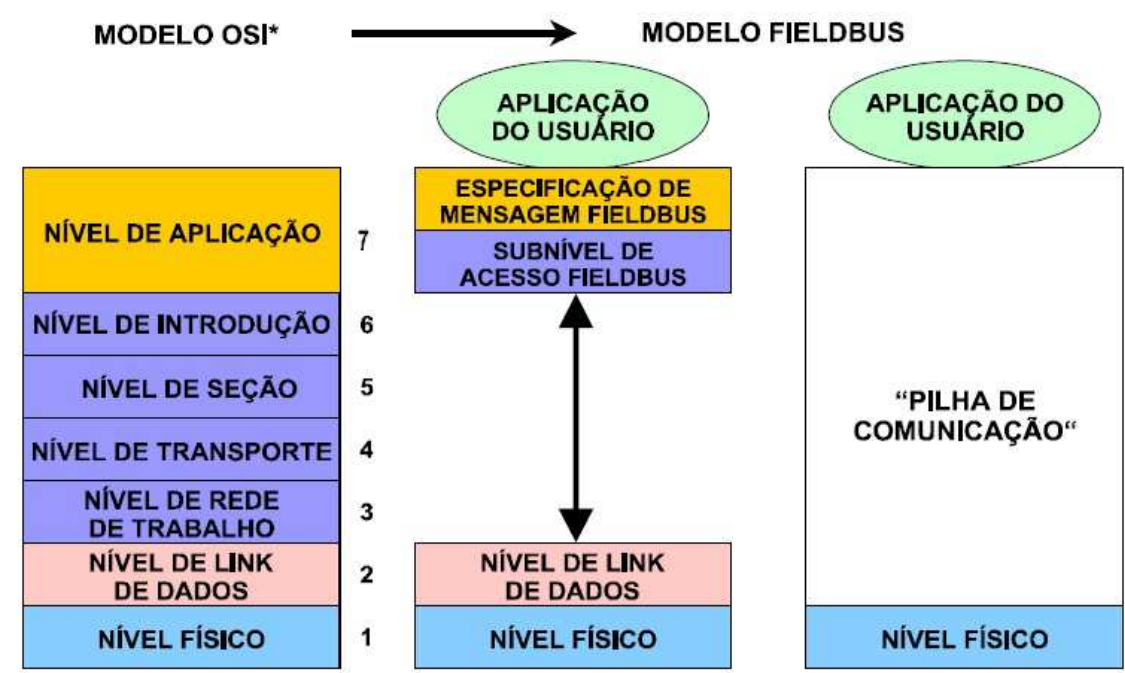

*A aplicação do usuário não é definido pelo modulo OSI.

Figura 5 - Comparativo do FF com o modelo OSI

$\mathrm{O}$ meio físico da rede $\mathrm{H} 1$ é um par trançado blindado. A alimentação e a comunicação se dão pelo mesmo par, necessitando de no mínimo $9 \mathrm{~V}$ no terminal do equipamento para energizá-lo e recomenda-se que esta tensão seja maior que $10 \mathrm{~V}$ e suficiente para manter um sinal íntegro de comunicação (0.75 Vpp a $1 \mathrm{Vpp})$, levando-se em conta a perda de tensão no cabo, o consumo total do barramento, e outros fatores. O comprimento da fiação pode chegar a $1900 \mathrm{~m}$, sendo que até 4 repetidores podem ser usados, atingindo $9600 \mathrm{~m}$.

A rede HSE baseia-se na mesma camada física Ethernet. Diversos fabricantes oferecem equipamentos específicos para aplicações industriais, seja com faixa de temperatura adequada $\left(-40\right.$ a $\left.85^{\circ} \mathrm{C}\right)$, seja com funções específicas para comunicação de dados em tempo real.

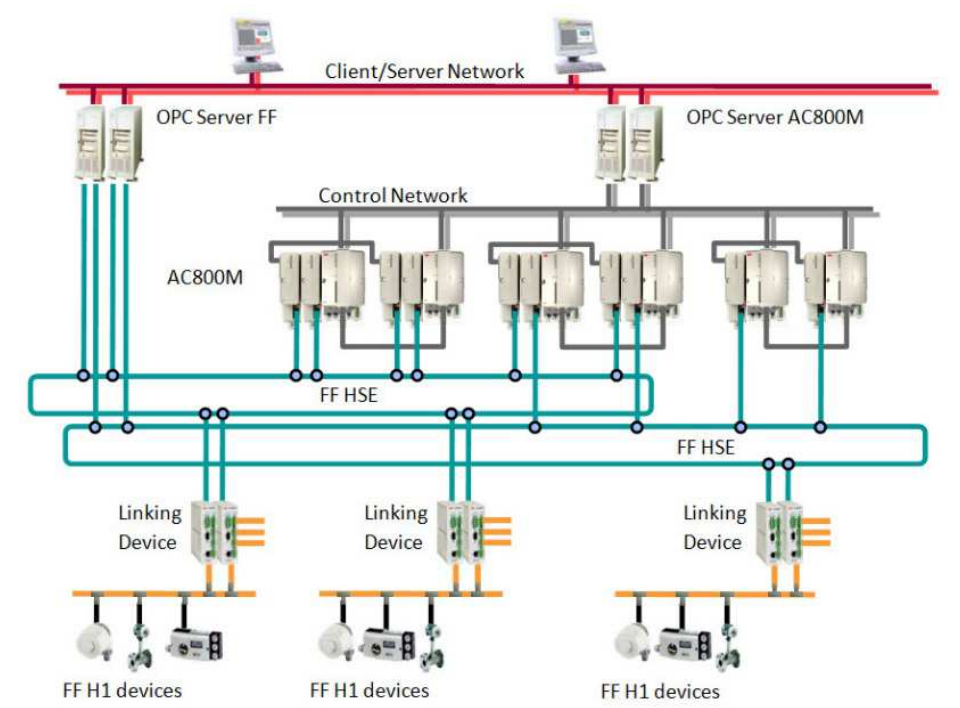

Figura 6 - Exemplo de infraestrutura FF com equipamentos ABB

\section{ANÁLISE COMPARATIVA DOS PROTOCOLOS}

Com o intuito de facilitar a comparação entre os protocolos, foi gerado a tabela 1 que compila as principais características técnicas de cada protocolo. 
Tabela 1 - Comparativo entre os protocolos

\begin{tabular}{|c|c|c|c|}
\hline Parâmetro & Profibus & $\begin{array}{l}\text { Fieldbus } \\
\text { Foundation }\end{array}$ & WirelessHART \\
\hline Tipo de rede & $\begin{array}{l}\text { DP: Device Bus; } \\
\text { PA: Rede de } \\
\text { Controle } \\
\text { Processo; }\end{array}$ & Rede de Controle & Rede de Controle \\
\hline $\begin{array}{l}\text { Estrutura } \\
\text { Física }\end{array}$ & $\begin{array}{l}\text { DP - RS485, Fibra } \\
\text { PA - IEC61158 }\end{array}$ & IEC61158 & - \\
\hline Topologia & Barramento, Estrela, Anel & Barramento, Estrela & Mesh \\
\hline $\begin{array}{l}\text { Distância } \\
\text { máxima do s } \\
\text { cabos }\end{array}$ & $\begin{array}{l}\text { DP: } 93,75 \text { Kbps ou menos }-> \\
1200 \text { metros } \\
500 \text { Kbps }->400 \text { metros } \\
1,5 \mathrm{Mbps}->200 \text { metros } \\
\text { Acima de } 1,5 \mathrm{Mbps}->100 \\
\text { metros } \\
\text { PA: } 1900 \text { metros }\end{array}$ & $\begin{array}{l}1900 \text { metros. } \\
\text { Pode ser estendido } \\
\text { para } 9500 \mathrm{~m} \text { com o uso } \\
\text { de até } 9 \text { repetidores. }\end{array}$ & $\begin{array}{l}\text { Depende do arranjo } \\
\text { dos escravos, a } \\
\text { distância máxima } \\
\text { recomendada entre os } \\
\text { escravos é de } 300 \\
\text { metros. }\end{array}$ \\
\hline $\begin{array}{l}\text { Método de } \\
\text { comunicação }\end{array}$ & $\begin{array}{l}\text { DP: ponto-a-ponto, multicast } \\
\text { ou mestre-escravo cíclico } \\
\text { (usando token passing } \\
\text { sequence); } \\
\text { PA: } \\
\text { Publisher/subscriber, Event, } \\
\text { ambos scheduled and } \\
\text { onscheduled } \\
\text { communications; }\end{array}$ & $\begin{array}{l}\text { Client/server, } \\
\text { Publisher/subscriber, } \\
\text { Event, ambos } \\
\text { scheduled and } \\
\text { onscheduled } \\
\text { communications }\end{array}$ & $\begin{array}{l}\text { TDMA, token-passing, } \\
\text { Smart Data Publishing }\end{array}$ \\
\hline Usabilidade & $\begin{array}{l}\text { DP: PLC, Remote I/O, } \\
\text { Variable Speed Drivers, } \\
\text { comunicação I/O remota; } \\
\text { PA: controle de processos. }\end{array}$ & $\begin{array}{l}\text { Controle de processos } \\
\text { (analógicos e } \\
\text { discretos) }\end{array}$ & Controle de Processos \\
\hline $\begin{array}{l}\text { Fonte de } \\
\text { Alimentação }\end{array}$ & $\begin{array}{l}\text { PA: Geralmente alimentado } \\
\text { via barramento. } \\
\text { DP: alimentação separada } \\
\text { do barramento de } \\
\text { comunicação }\end{array}$ & $\begin{array}{l}\text { Geralmente } \\
\text { alimentado via } \\
\text { barramento }\end{array}$ & Geralmente Baterias. \\
\hline \multirow[t]{3}{*}{ Tipo de Fiação } & $\begin{array}{l}\text { DP: Par trançado blindado, } \\
\text { \#22 AWG; } \\
\text { PA: Par trançado blindado, } \\
\text { \#18AWG. }\end{array}$ & $\begin{array}{l}\text { Tipo A - Par trançado } \\
\text { protegido, \#18AWG; } \\
\text { Tipo B - Multi-par } \\
\text { trançado com } \\
\text { proteção, \#22AWG; }\end{array}$ & Sem cabo. \\
\hline & & $\begin{array}{l}\text { Tipo C }- \text { Multi-par } \\
\text { trançado sem } \\
\text { proteção, \#26AWG; }\end{array}$ & \\
\hline & & $\begin{array}{l}\text { Tipo D - Multi-core } \\
\text { sem par trançado e } \\
\text { com alta proteção, } \\
\text { \#16AWG. }\end{array}$ & \\
\hline Terminadores & $\begin{array}{l}\text { No início e fim } \\
\text { seguimento }\end{array}$ & $\begin{array}{l}\text { No início e fim do } \\
\text { seguimento }\end{array}$ & Sem necessidade \\
\hline
\end{tabular}


Em seguida apresentamos as principais vantagens e desvantagens de cada protocolo.

Vantagens WirelessHART:

- Baseado no protocolo HART, portanto existe compatibilidade com instrumentos de campo, aplicações e ferramentas HART já instalados;

- A camada física e de controle ao meio de acesso (MAC) são baseados no padrão IEEE 802.15.4-2006;

- Utiliza encriptação e autenticação para garantir que a comunicação seja segura;

- Simples de implantar e operar;

- Recupera-se de falha automaticamente.

- Auto organizável;

- Potência de transmissão ajustável;

- Economia incrível no custo com cabos.

Vantagens Fieldbus Foundation:

- Controle no nível de Planta (campo);

- Pode ser utilizado em instalações intrinsecamente segura;

- Possibilidade de realizar comandos de calibração e parametrização remotos;

- Interoperabilidade entre fabricantes, pelo protocolo temos os Blocos de função predefinido podendo assim utilizar por exemplo a mesma parametrização de um bloco "Al" independente do fabricante;

- Vasta gama de diagnósticos do instrumento, o que pode ser usado no gerenciamento de ativos;

- É possível implementar a redundância de alimentação.

Vantagens Profibus:

- Altas taxas de velocidade do DP, podendo chegar até 12Mbps;

- Pode ser utilizado em área intrinsecamente seguras, fazendo uso da tecnologia PA;

- Integração dos instrumentos PA em redes DP, através do uso de gateways;

- Host disponíveis para a maioria dos PLC's e SDCD's do mercado, por exemplo: ABB 800xa;

- Mais de dois mil equipamentos certificados.

Desvantagens do Profibus

- Necessário um cuidado imenso com preparação de infraestrutura;

- Não permite controle em nível de campo.

\section{WirelessHART}

- Têm-se que investir tempo para projetar o arranjo dos instrumentos para que cada nó da rede tenha "visada" para no mínimo três outros;

- Necessidade de repor as baterias;

- Não indicado para controles críticos, uma vez que diminuindo o intervalo utilizado entre as transmissões, diminui também a vida útil da bateria. 
Desvantagens do Fieldbus Foundation

- Como todos os instrumentos utilizam o mesmo canal de comunicação, dependendo da quantidade de instrumentos "pendurados" em um seguimento, o macro-cycle torna-se muito grande, impossibilitando um controle preciso;

- Necessário um cuidado imenso com preparação de infraestrutura;

- A maior dificuldade é a mão de obra especializada para a manutenção da rede e dos dispositivos;

\section{CONCLUSÃo}

A escolha do protocolo a ser utilizado é muito importante para que seja obtido um desempenho satisfatório na operação de redes fieldbus.

Utilizando a experiência da equipe, obtida com a implantação de diversos projetos de automação nos últimos anos, pode-se afirmar que as empresas de engenharia preocupam-se pouco em analisar os fatores positivos e negativos do uso destes protocolos durante a fase de especificação do projeto, muitas vezes considerando somente o quanto será economizado em cabos com a adoção de um protocolo ou outro, como parâmetro decisivo para sua adoção em projetos.

Uma ação saudável para projetos de fieldbus futuros pode ser a adoção de vários destes protocolos na mesma planta, utilizando dos seus pontos fortes para que obter uma performance produtiva.

\section{REFERÊNCIAS}

1 GUEDES, L.A. Classificação das redes para automação industrial. 2005. [Acesso em 30 mar. 2009]. Disponível em: <http://www.dca.ufrn.br/ affonso /DCA0447/aulas/rai_cap3_part1.pdf>.

2 PINTO, Pedro. Redes - Sabe o que é o modelo OSI? [Acesso em 10.Mar.2015]. Disponível em: <http://pplware.sapo.pt/tutoriais/networking/redes-sabe-o-que-e-omodelo-osi/>

3 MOSSIN, E. (2012). Diagnóstico Automático de Redes Profibus. Tese (Doutorado). Escola de Engenharia de São Carlos, USP.

4 Mecatrônica Atual. [Acessado em 10.Mar.2015]. Disponível em: http://www.mecatronicaatual.com.br/educacao/1866-caractersticas-do-protocoloprofibus-e-sua-utilizao-em-reas-classificadas

5 SONG, Jianping et al. WirelessHart: Applying Wireless Technology in Real-Time Industrial Process Control. IEEE Real- Time end Embedded Technology and Applications Symposium, Austin, 2008.

6 JUNIOR, Lellis do Amaral Campos. WirelessHart - Tecnologia Wireless aplicada a instrumentos de campo. Revista Controle \& Instrumentação, no144, pp. 74 -78. Março 2009

7 SEABRA, Hugo Viana Magalhães; LUGLI, Alexandre Baratella. Redes sem fio para Automação Industrial: Estudo e Aplicação. 